\title{
Outcomes That Matter Most to Young Adults Diagnosed with Cancer: A Qualitative Study
}

\author{
Paul Guzik, DO, ${ }^{1, *}$ Jennifer A. McKinney, $\mathrm{PhD},{ }^{2,5,{ }^{*}}$ Christopher Ulack, $\mathrm{PhD},{ }^{3}$ Joel Suarez, $\mathrm{PhD}{ }^{3}$ \\ Victoria Davis, $\mathrm{PhD},{ }^{3}$ Elizabeth Teisberg, $\mathrm{PhD},{ }^{3,4}$ Scott Wallace, JD, MBA, ${ }^{3,4}$ \\ S. Gail Eckhardt, MD, ${ }^{2,5}$ and Anna Capasso, MD, $\mathrm{PhD}^{1,2,5}$
}

Purpose: The purpose of this study is to provide insight for improvement in care for young adults diagnosed with cancer (YADC), by identifying underemphasized outcomes that strongly matter to YADC and the gaps in care that may limit achieving these outcomes for this unique and vulnerable population.

Methods: Twenty-seven YADC, ages 25-39, participated in unstructured discussions focusing on topics relating to diagnosis, daily experiences living with cancer outside of the clinical setting, goals, concerns, and clinical care experience. Most participants engaged in group discussions using Experience Group methodology. Discussions were designed to collect information on three dimensions of health: capability, comfort, and calm (CCC). Data were coded using thematic analysis with NVivo software.

Results: Several themes were identified within the CCC framework: capability in terms of confronting mortality at a young age, losing youthful identity and control over major life course decisions, especially fertility, and debilitating side effects, comfort in terms of the lack of understanding from peers and family and the fear of cancer recurrence, and calm was discussed as the difficulty of making complex medical decisions, financial toxicity, and loss of clinical support in survivorship.

Conclusion: This research highlighted four care additions that are important for YADC: (1) concise and understandable education about their condition and treatment; (2) same-age support groups; (3) fertility support; and (4) better care transitions for life after cancer. These findings emphasize the importance of creating a collaborative, multidisciplinary care team and a holistic approach with care innovations that support clinicians to meet the unique needs of YADC.

Keywords: qualitative study, health outcomes, clinical support, standard of care, patient-centered care, care design

\section{Introduction}

N The United States, $\sim 80,000$ young adults aged 20 $39^{1}$ are diagnosed with cancer each year. ${ }^{2}$ Cancer is the fourth leading cause of death for this age group, claiming $\sim 9000$ lives each year in the United States alone. ${ }^{2}$ There are many types of cancer that can occur in young adults. ${ }^{1}$ Regardless of type, receiving a cancer diagnosis disrupts a vital and dynamic period of development and growth for young adults, many of whom may be in the early stages of navigating new-found independence from parents and embarking on life with limitless possibilities or may be focused on building and cultivating families, social networks, and careers. ${ }^{3}$ A life-threatening disease in early adult life changes one's perspective on hopes and dreams in ways that need intentional support.

Young adults diagnosed with cancer (YADC) face several unique obstacles in comparison to both their same-age healthy peers and other age cohorts with cancer. For example, while a cancer diagnosis can result in an overwhelming financial burden at any age, young adults tend to have less financial stability than older adults. Furthermore, despite an observed increase in insurance coverage by young adults since the full implementation of the Affordable Care

\footnotetext{
${ }^{1}$ Department of Internal Medicine, ${ }^{2}$ Livestrong Cancer Institutes, ${ }^{3}$ Value Institute for Health \& Care, ${ }^{4}$ Department of Medical Education, and ${ }^{5}$ Department of Oncology, The University of Texas at Austin Dell Medical School, Austin, Texas, USA.

*These authors contributed equally to this work.

(C) Paul Guzik et al. 2020; Published by Mary Ann Liebert, Inc. This Open Access article is distributed under the terms of the Creative Commons Attribution Noncommercial License [CC-BY-NC] (http://creativecommons.org/licenses/by-nc/4.0/) which permits any noncommercial use, distribution, and reproduction in any medium, provided the original author(s) and the source are cited.
} 
Act, cancer care remains a massive and unexpected expense. ${ }^{4}$ Younger cancer patients also tend to receive more aggressive treatment compared to older patients resulting in an even greater financial burden. ${ }^{5}$

Often, young adult survivors must manage debilitating side effects of treatment while also struggling to find a new identity and purpose in life after cancer. Therefore, it is disheartening to find that many YADC historically reported unmet physical and psychosocial needs both during and after their cancer care. ${ }^{6-8}$ A 2011 meta-analysis found the "late effects" of cancer treatment (including secondary malignancies, physical limitations, psychosocial difficulties, and fertility problems) more common in adolescents and young adults compared to older or younger cancer survivors. ${ }^{9}$ Other studies have shown young adults reporting a higher incidence of major physical concerns, especially chronic fatigue ${ }^{10}$ and fertility problems. ${ }^{11}$ In one study, as many as $75 \%$ of adolescents and young adults stated that they were not provided with information on support groups. ${ }^{8}$

This study aimed to engage YADC in discussions outside of a clinical setting to allow participants the freedom to openly discuss daily experiences, concerns, and goals, with the purpose of understanding obstacles and unmet needs that were previously unarticulated. To achieve this, we used Experience Group (EG) methodology ${ }^{12}$ and the capability, comfort, and calm (CCC) framework of health outcomes, ${ }^{13}$ developed to improve clinicians' understanding of the outcomes that matter most to patients and the experiences outside of clinical care that undermine or enhance those outcomes. EG discussions encourage frank and open discussion among patients who faced similar health challenges. In EG research with patient groups across dozens of types of health challenges, researchers found that the outcomes that mattered most were robustly described in three dimensions of health that matter most to patients within the CCC framework: capability (ability to do the things that allow me to be me), comfort (reduction of physical or mental suffering), and calm (the experience of a healing embrace while living with cancer, free from the unwarranted stress and chaotic experiences often associated with getting treatment). ${ }^{12,14-16}$ Using the CCC framework to organize our insights, we identified several themes that are important for YADC to achieve the outcomes of these dimensions, as well as identified gaps and obstacles for patients in improving these outcomes. Designing YADC care that addresses these gaps and obstacles will also support clinicians who now muster extra effort and empathy to see and meet YADC needs. The findings from this study emphasize the need for care solutions that better fit into the lives of young adults and can be used to improve the design and delivery of care to this vulnerable population.

\section{Methods}

\section{Study participants}

Participants $(n=27)$ ranged in age from 25 to 39 (22 females, 5 males). Twenty-two participants spoke English as a primary language; four participants spoke Spanish; and one participant spoke both English and Spanish (Table 1). Cancer diagnoses included breast cancer, cervical cancer, Hodgkin's disease, non-Hodgkin lymphoma, leukemia, multiple myeloma, and rhabdomyosarcoma. Some participants were in
TABle 1. Demographic INFORMATION

\begin{tabular}{ll}
\hline Age & Range: $25-39$ years old \\
& Average: 32.24 years old \\
Gender & Female: $22 / 27(81.5 \%)$ \\
& Male: $5 / 27(18.5 \%)$ \\
Language & English: $22 / 27(81.5 \%)$ \\
& Spanish: $4 / 27(14.8 \%)$ \\
& Bilingual: $1 / 27(3.7 \%)$ \\
\hline
\end{tabular}

treatment at the time of the study, and others were in remission. Participants were compensated with a grocery store gift card. This study was determined to be IRB exempt based on 45 CFR 46.101(b)(2) by the Office of Research Support and Compliance at the University of Texas at Austin.

\section{Session format and data collection}

Seven EG sessions $(n=21)$ and six individual sessions $(n=6)$ were conducted with the YADC participants. All individual sessions were audio recorded and transcribed verbatim. Four of the EG sessions were audio recorded and transcribed verbatim, while data from the remaining three sessions were recorded manually by a nonfacilitating researcher. The majority of discussions were conducted in English, and four individual discussions were conducted in Spanish.

Individual sessions were conducted with participants who could not attend one of the group sessions. Small group discussions (or EG sessions), consisting of 3-8 study participants, were preferred because the dynamic among the participants adds a supportive atmosphere and opens conversation topics that session leaders may not anticipate and allows for exploration of participants' shared experiences outside of the clinic environment and discussions of deep issues of concern beyond parking, waiting rooms, or hospitality. Discussions lasted 60-90 minutes, and topics were posed as open-ended questions (Supplementary Appendix SA1) to avoid priming participants' answers.

\section{Data analysis}

Researchers debriefed for $\sim 30$ minutes after each session to discuss key experiential themes the participants articulated, as well as identify the goals and outcomes that participants discussed, and the obstacles or unmet needs that may slow progress to those outcomes. Sessions were transcribed and underwent thematic coding using NVivo software to further analyze and organize the data within the CCC framework.

\section{Results}

Thematic coding and analysis of the data yielded the following themes, which were organized into the CCC framework. Additional participant quotations are included in Supplementary Table S1.

\section{Capability}

Having the ability to do the things that support one's identity and normal life, as the individual defines, is the essence of regaining capability. 
Confronting mortality as a young adult

$$
\begin{aligned}
& \text { "Nobody's really come to terms with their [own] mortal- } \\
& \text { ity... at that point." }
\end{aligned}
$$

With a cancer diagnosis, the capability to lead one's normal life starts with survival. Many participants, regardless of their cancer type, said that confronting the reality of dying young, as opposed to planning life during and after treatment, was their initial reaction to their diagnosis. Participants stated that they stopped assuming they were going to live a long life. Nevertheless, several participants felt that understanding this truth at a young age freed them to live a different life. It gave them a new appreciation for life and reminded them to not take things for granted.

\section{Loss of youthful identity and control on life}

\footnotetext{
"For me the hardest thing was when my hair started falling out...When it started my hair was so long. [...] My hair was, just, a part of me. It was something that was very important for my identity as a woman."
}

Several participants felt as though their diagnosis and the effects of treatment had stripped them of their youthful identity and sense of invincibility. One young male participant illustrated this shift by describing training for a marathon just before diagnosis, and then, only a short time after, using a Fitbit to limit his steps due to fatigue. This loss of youthful identity was associated with changes in physical appearance (e.g., hair loss), a decrease in the ability to engage in physical activities, and the need for constant medical care.

Participants also felt a sudden loss of control over their lives. Cancer controlled how much they worked or could go to school, when they were hungry, how they looked or felt, where they had to be, and what they could or could not do. The loss of control is particularly devastating for young adults who are learning how to navigate their new-found independence and provoked several emotions for participants from anger and frustration to hopelessness and sadness.

\section{Debilitating treatment side effects, especially infertility}

\footnotetext{
“...that fertility piece is actually one of the biggest pieces, and not everybody wants to be a mother, not everybody has that interest or even biologically that ability, but that is such a huge decision to have to make."
}

Side effects from medical and surgical treatment can be debilitating for anyone; however, the impact can be magnified in young adults who are at a stage when physical limitations seem unfamiliar and who may be building a career or raising young children. Participants' number one priority during treatment was survival, but in retrospect, YADC wanted to be more informed about side effects when considering treatment options. Many participants expressed the difficulty of returning to school or work due to physical ailments, as well as cognitive impairment (Table 2).

Regardless of reproductive age, gender, and relationship status, fertility remained a common source of stress. Several women associated infertility with a "loss of purpose" and "loss of who [they] are as a human being." Some of the
Table 2. Most Debilitating Side Effects IN Young Adults

Side effect

Fertility problems

Fatigue/chronic pain

Psychological effects (depression, anxiety, post-traumatic stress disorder)

Hair loss

Memory problems ("chemo brain")

"Early" menopause-like symptoms

YADC had not even started to consider having children before their diagnosis, yet felt intense pressure to make decisions about fertility preservation even if they were single, and the idea of becoming sterilized in one's 20s or 30s to save their life can be especially devastating.

\section{Comfort}

Reducing physical and mental suffering is a primary purpose of health care. YADC face a frightening and unusual situation, occurring at a time in their lives when independence is often highly valued.

\section{Lack of understanding from peers and family}

\footnotetext{
"I can't turn to my friends or family because they're not going through this and although they're supportive, the concept of being with people and not having to explain everything all the time is really [important]."'
}

YADC found it difficult to find other people with whom they could relate and therefore felt lonely and distant from family, friends, and even older adults with cancer. Many found it difficult to communicate with friends and family about their diagnosis and felt that they were treated differently following their diagnosis, although most did not want to be. In addition, several participants felt they were stigmatized for having cancer as a young adult as though they had done something wrong, which caused participants to avoid discussions about cancer and, in several cases, hide their diagnosis altogether. In addition, when mixed-age support groups were offered to YADC, they felt out of place among the older adults with cancer who were invariably part of the mix.

\section{Fear of recurrence}

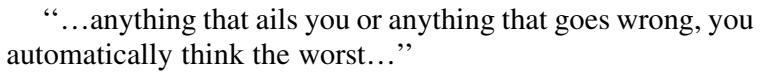

Although the fear of recurrence is not unique to YADC, it is magnified by their youth. For many participants, post-treatment life was marked by a sense of aimlessness and loss of direction. Many often found themselves wondering, "now what?" As appointments and testing became less frequent during remission, patients worried that potential symptoms of recurrence, such as unexplained aches or pains, were missed. Participants also expressed an increase in anxiety in anticipation of surveillance laboratories, imaging, and appointments, feeling as though there were very few resources to help reassure them outside of the appointment 
itself. Participants felt a preappointment phone call discussing the agenda for the visit and answering any questions would have helped calm their worries.

The psychological implications of cancer were as significant as the physical side effects mentioned above. Numerous participants mentioned having post-traumatic stress disorder after surviving cancer. Many participants initially resisted counseling services, but those who did utilize therapy felt as though they benefited immensely, while some did not realize they needed therapy until receiving it well after finishing treatment. Participants insisted that psychological counseling be automatically offered at the moment of diagnosis, rather than having an emotional crisis triggering a patient to seek psychological support.

\section{Calm}

Healing is supported by feeling the embrace of care. Equally important to the inpatient experience is the patient feeling enabled and supported in one's life and health care journey outside of the clinical environment.

\section{Difficulty making complex medical decisions}

“...when your oncologist is like, you get to choose... I felt paralyzed, because I was like, I don't have any medical background. I don't know what to do to make this not come back.",

Aside from a few nurses and health care workers that were included in this study, most of the participants had no medical background. Participants felt intense pressure to learn the complexities of cancer care in a short period of time to make important decisions about their treatment. Some participants expressed appreciation for the opportunity to decide on elements of their own treatment, yet still found it overwhelming to be responsible for making decisions about their own treatment without the requisite knowledge (or time to acquire and truly understand such knowledge) that would enable them to feel comfortable about their decision. Several participants described the days and weeks after diagnosis as stepping into a "whirlwind" of information, uncertainty, and decision-making.

\section{Financial toxicity}

“...you're just starting your life, and then you basically get pushed into a hole and they keep throwing dirt on top of you, and you're just trying to dig yourself out of this hole. You can't get out because all this debt keeps being thrown on you."

All participants had medical insurance or qualified for Medicaid, and some had designated "cancer coverage," yet participants still felt the overwhelming costs of cancer care. Because of their stage in life, many of the YADC participants did not have much - or any-money set aside for emergencies. Once they were in remission, several participants realized that their life goals and family plans (e.g., owning a home or having children) were derailed as a result of their financial situation. Participants universally spoke of the dire need for direct financial assistance, including help negotiating with insurance companies and navigating their complex policies, and resources for financial support from public and private institutions.

\section{Loss of clinical support after treatment}

“...Afterwards, I was in normal life, and it kind of hits you what you've been through and how you're feeling. I think I struggled more afterwards than I did during it and during the diagnosis."

Young adults felt that the transition from treatment into survivorship was just as important as the transition from health to a cancer diagnosis, yet the former was often overlooked. Although clinical support was abundant during treatment, participants felt both physically and emotionally abandoned by the health care system in survivorship. Several participants were left to find a primary care provider, who often had little knowledge of their malignancy or how to manage their medications and treatment-related side effects. In addition, many participants felt a sense of guilt when they asked questions or complained about how they were feeling during remission because they feared it betrayed a lack of appreciation for having survived cancer.

\section{Discussion}

A cancer diagnosis, treatment, and survivorship or loss can be debilitating, burdensome, and traumatic for a patient of any age and their caregivers. Because of the unique challenges YADC face throughout their cancer journey, their care may need innovative approaches that are different compared with other patients. Improved outcomes for YADC could involve incremental change in current care to specifically meet the needs of young adults or could entail the creation of a focused, comprehensive YADC care program. These changes in care processes would benefit not only YADC but also clinicians who now attend to YADC needs by devoting extra time and empathy that are required because the normal processes are not designed for YADC needs.

This study used the CCC framework to focus the outcomes that matter most to people seeking health care. ${ }^{12,14,15}$ Efforts to improve care often focus on patient satisfaction and improvements in hospitality, waiting times, or parking, which are less essential than healing, reducing suffering, and getting one's life back on track. The insights from this study point to opportunities for changes in care delivery to improve the health and well-being that patients achieve.

\section{Improving capability to lead one's normal life}

After treatment, YADC want to regain aspects of their youthful identity in an effort to return to "normal life." Regaining the ability to engage in physical activity, socialize with peers, work, or go to school allows YADC to reclaim their identity and recapture a sense of control over their lives. Physicians often underestimate the impact of treatmentrelated fatigue on quality of life, and many patients fear that reporting fatigue may cause providers to pursue less aggressive cancer treatment; ${ }^{10}$ however, providing care that focuses on living well and includes age-appropriate support such as nutrition and exercise, even during treatment, can be enabling for young adult patients.

In addition, this study highlighted a need for more comprehensive information for managing treatment-related side effects, especially infertility. Unfortunately, cancer and its treatments can impact fertility in both men and women. In 
this study, and others, participants felt that the fertility discussion with their care providers was incomplete and often ill-timed; patients want a more comprehensive discussion of risks to fertility and fertility preservation options. ${ }^{11}$ Providing explicit and early counseling regarding fertility issues and options for young adults, either with an on-site fertility specialist or referral, can affirm their life after treatment, whereas not providing this service can diminish hope.

\section{Improving comfort during and after cancer care}

YADC felt isolated and lonely among healthy peers and family, but also among older adults with cancer. YADC expressed a desire for support groups but felt left out in those that were offered because they were the youngest person in the group by several decades. Young adults also felt like their family and friends would significantly benefit from a caregiver support group. Because providers did not offer caregiver support, several participants felt like they became responsible for providing that support for their parents, contributing to more stress on the patient. In addition to support groups, participants advised other YADC to seek therapy. Providers can mitigate stress on the patient by enhancing their comfort both in and out of the clinic. Examples include resources for same-age support groups for patients and caregivers, redesigning waiting rooms to gathering spots for patient-patient interaction, or integrating counseling as a normal part of treatment.

\section{Improving calm during the embrace of care}

YADC would like a multidisciplinary team to educate and support them as they make difficult medical decisions. Often times, YADC are no longer relying on their parents to make decisions, may be caring for small children or their own aging parents, and have limited sources of support during this hectic time. Having a patient advocate on the clinical team whose sole purpose is to answer questions and provide resources could help empower patients to engage in shared decision making. In addition, the need for financial assistance is desired for YADC who likely have limited savings or experience with insurance companies and medical debt. A designated advocate to speak with insurers on the patient's behalf would greatly help YADC with navigating their care. Investing in this type of patient advocate would also free clinical staff from these tasks and allow them to care for the patient in other ways.

Many participants also felt a lack of clinical support after treatment, describing the care they received as fragmented. Although survivorship care is now offered in many places, YADC require additional resources to help them through this transition and beyond. YADC want a plan, or at least clarity, on post-treatment life to mitigate the confusion and isolation they felt. In short, the medical team must attend to delivering information in a more comprehensible way, especially during transitional periods.

\section{Limitations}

The main limitation of our study was the participant population. We would like to expand this study to increase the sample size, as well as include participants from more diverse backgrounds, including different ethnicities, cultures, coun- tries, and health care systems, as it would be of interest to compare and contrast how these differences influence what participants view as the most important patient outcomes.

\section{Conclusion}

The purpose of cancer care is to save lives and improve health and well-being. Success with patient-centric care requires understanding the patient perspective. By examining the experiences of YADC during treatment and survivorship, this qualitative study provides a preliminary evaluation of what matters most to this vulnerable population. Discussions with 27 YADC identified 8 important themes that impact the outcomes of capability, comfort, and calm during and after cancer care.

Clinical teams can work to address these issues in many ways. Four steps within the stride of many clinical teams are as follows: (1) addressing the need for better care transitions, especially in dealing with debilitating side effects during survivorship; (2) early education and support regarding fertility preservation; (3) improved emotional support through same-age support groups, caregiver support groups, and integrated therapy; and (4) creating a patient advocate to help navigate difficult medical decisions and assist with insurers or financial difficulties. The findings of this study emphasize the importance of creating a collaborative multidisciplinary approach to cancer care that supports clinicians to address all aspects of care to improve the outcomes that matter most to YADC. How to provide this information so that it is accessible to patients and members of the care team in line with our results is the focus of future studies.

\section{Acknowledgments}

The authors of this article thank the University of Texas at Austin Dell Medical School, Livestrong Cancer Institutes, and the Value Institute for Health \& Care for their support in this research endeavor.

\section{Author Disclosure Statement}

No competing financial interests exist.

\section{Funding Information}

This research was supported, in part, by CPRIT grant RR160093 (S.G.E. "CPRIT SCHOLAR IN CANCER RESEARCH"). This research was supported, in part, by the Cullen Trust for Higher Education.

\section{Supplementary Material}

Supplementary Appendix SA1

Supplementary Table S1

\section{References}

1. Types of cancers that develop in young adults: American Cancer Society; 2020. Accessed January 1, 2019 from: https://www.cancer.org/cancer/cancer-in-young-adults/cancersin-young-adults.html

2. Key statistics for cancers in young adults: American Cancer Society; 2020. Accessed January 1, 2019 from: https://www .cancer.org/cancer/cancer-in-young-adults/key-statistics.html 
3. De P, Ellison LF, Barr RD, et al. Canadian adolescents and young adults with cancer: opportunity to improve coordination and level of care. CMAJ. 2011;183(3): E187-94.

4. Spencer DL, McManus M, Call KT, et al. Health care coverage and access among children, adolescents, and young adults, 2010-2016: implications for future health reforms. J Adolesc Health. 2018;62(6):667-73.

5. Smith TJ, Penberthy L, Desch CE, et al. Differences in initial treatment patterns and outcomes of lung cancer in the elderly. Lung Cancer. 1995;13(3):235-52.

6. Hall AE, Boyes AW, Bowman J, et al. Young adult cancer survivors' psychosocial well-being: a cross-sectional study assessing quality of life, unmet needs, and health behaviors. Support Care Cancer. 2012;20(6):1333-41.

7. Lie NK, Larsen TMB, Hauken MA. Coping with changes and uncertainty: a qualitative study of young adult cancer patients' challenges and coping strategies during treatment. Eur J Cancer Care (Engl). 2018;27(6):e12743.

8. Keegan TH, Lichtensztajn DY, Kato I, et al. Unmet adolescent and young adult cancer survivors information and service needs: a population-based cancer registry study. J Cancer Surviv. 2012;6(3):239-50.

9. Woodward E, Jessop M, Glaser A, Stark D. Late effects in survivors of teenage and young adult cancer: does age matter? Ann Oncol. 2011;22(12):2561-8.

10. Campos MP, Hassan BJ, Riechelmann R, Del Giglio A. Cancer-related fatigue: a practical review. Ann Oncol. 2011;22(6):1273-9.
11. Quinn GP, Vadaparampil ST. More research, more responsibility: the expansion of duty to warn in cancer patients considering fertility preservation. Am J Obstet Gynecol. 2013;209(2):98-102.

12. Teisberg E, Wallace S, O'Hara S. Defining and implementing value-based health care: a strategic framework. Acad Med. 2020;95(5):682-5.

13. Wallace S, Teisberg EO. Capability, comfort, and calm: designing health care services for excellence and empathy. Austin, TX: University of Texas at Austin; 2017.

14. Liu TC, Bozic KJ, Teisberg EO. Value-based Healthcare: person-centered Measurement: focusing on the Three C's. Clin Orthop Relat Res. 2017;475(2):315-7.

15. Wallace S, Teisberg EO. Measuring what matters: connecting excellence, professionalism and empathy. Brain Injury. 2016;12(2):12-4.

16. Porter ME, Teisberg EO. Redefining health care: creating value based competition on results. Boston, MA: Harvard Business School Press; 2006.

Address correspondenceto: Paul Guzik, DO

Department of Internal Medicine

The University of Texas at Austin Dell Medical School 1500 Red River Street Austin, TX 78701

USA

Email: paul.r.guzik@gmail.com 